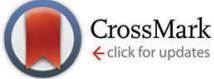

Cite this: J. Mater. Chem. C, 2016 4, 10786

Received 30th July 2016,

Accepted 29th October 2016

DOI: $10.1039 / \mathrm{c} 6 \mathrm{tc} 03260 \mathrm{e}$

www.rsc.org/MaterialsC

\section{Responsive aggregation-induced emissive supramolecular gels based on bis-cyanostilbene derivatives $\dagger$}

\author{
Yao Ma, ${ }^{a}$ Massimo Cametti, ${ }^{b}$ Zoran Džolić ${ }^{\star c}$ and Shimei Jiang ${ }^{{ }^{a}}$
}

\begin{abstract}
In aromatic solvents, V-shaped bis-cyanostilbene derivative 1 forms stable and emissive gels which are capable of responding to light and, selectively, to TFA via a gel-to-sol transformation.
\end{abstract}

Low-molecular-weight organic gelators (LMWOGs) have received considerable attention in supramolecular chemistry and materials science due to their unique properties and a wide range of potential applications. ${ }^{1,2}$ Apart from basic studies on gel formation and structural morphology, extensive efforts have been devoted to the investigation of functional and stimuli responsive gels. ${ }^{3}$ The preparation of smart soft materials able to react to external stimuli and translate them into a well-defined, controllable, and reversible macroscopic response represents one of the most fascinating and challenging goals of current research on gel materials. This explains the numerous works present in the literature, where the bulk shape and properties of supramolecular gels based on LMWOGs are found to be responsive to a range of external stimuli of chemical and physical nature. ${ }^{4}$ As to the latter kind, the use of light is of particular interest for it allows both spatial and temporal control of a specific reaction without requiring physical contact. ${ }^{5}$ Recently, processes like aggregation induced emission (AIE) or aggregation induced emission enhancement (AIEE) have been utilized in developing "stimuli-responsive fluorescent gels", which exhibit gel-to-sol phase transitions along with remarkable fluorescence modulation. ${ }^{6}$ The design of stimuli-responsive fluorescent gelators is very challenging and thus it has only rarely been reported. Indeed, molecules that maintain their fluorescence in the aggregated state are quite rare

\footnotetext{
${ }^{a}$ State Key Laboratory of Supramolecular Structure and Materials, Jilin University, 2699 Qianjin Avenue, Changchun 130012, P. R. China.

E-mail: smjiang@jlu.edu.cn; Fax: +86-431-85193421; Tel: +86-431-85168474

${ }^{b}$ Department of Chemistry, Materials and Chemical Engineering "Giulio Natta",

Politecnico di Milano, Via L. Mancinelli 7, 20131 Milano, Italy

${ }^{c}$ Rudjer Bošković Institute, Bijenička cesta 54, 10000 Zagreb, Croatia.

E-mail: zoran.dzolic@irb.hr

$\dagger$ Electronic supplementary information (ESI) available: Detailed synthetic procedures and characterization data, NMR spectra, UV/Vis and fluorescence spectra. See DOI: $10.1039 / \mathrm{c} 6 \mathrm{tc03260e}$
}

and more so those also capable of both gelation and stimuli responsiveness. Among the fluorescent organogels reported to date, only very few examples exhibit aggregation-induced emission (AIE) properties. In such cases, fluorophores that exhibit almost no fluorescence as discrete molecules in dilute solutions become highly emissive in an aggregated state. As a consequence of the reversible nature of the self-assembly process at the basis of supramolecular gelation, AIE gel materials may exhibit significant modulation of their emission upon external inputs. Although several AIE organogels have been reported in the past years, examples of a rational application of established design principles for obtaining stimuli-responsive supramolecular organogels are rare.

Recently, we have developed a class of effective LMOGs based on V-shaped cyanostilbene amide derivatives, which readily formed gels in various organic solvents and also exhibited interesting anion-binding properties and AIE behaviour. ${ }^{7}$ Herein, we present a detailed study on the V-shaped compound 1 (Fig. 1A) based on a 1,3,5 substituted benzene core decorated with two cyanostilbene units via an amidic bond and with a hydrophobic $\mathrm{C}_{8}$ tail. Compound $\mathbf{1}$ is capable of forming stable and emissive gels in aromatic solvents which, interestingly, can be transformed into solution in response to either light (365 nm) or its exposure to trifluoroacetic acid (TFA). The latter transformation is quite selective in terms of the acidic species involved. Moreover, at variance with other acid-responsive gel systems reported, ${ }^{8}$ no acid-base reaction seems to be the triggering event of the observed behaviour. In addition, the effect of the chain length has been taken into account as shorter and longer chain derivatives, $\mathbf{2}$ and 3, were also synthesized and tested.

The gelation ability of 1-3 was systematically investigated (Table S1 in the ESI $\dagger$ ). Compounds $\mathbf{1}$ and $\mathbf{2}$ were able to form gels in all the tested aromatic solvents. In particular, compound $\mathbf{1}$ showed the best gelation performance, showing a lower critical gelation concentration ( $\mathrm{cgc}$ ). In general, $\operatorname{cgc}$ of 1 and 2, depending on the solvents, varied in the range of $2.9-8.3$ and $3.5-10.0 \mathrm{mg} \mathrm{mL}^{-1}$, respectively. The obtained gels were stable and could endure ambient conditions for several weeks. In contrast, compound 3, having a shorter chain length, does not form gels with any of the 
A
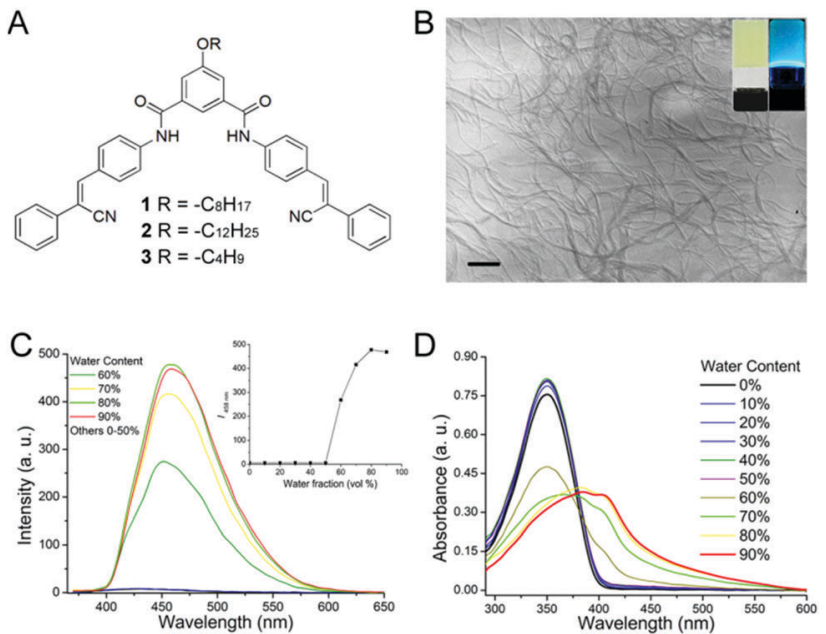

Fig. 1 (A) Molecular formulae of the $V$-shaped bis-cyanostilbene derivatives 1-3. (B) TEM image of toluene gel 1 (scale bar $=500 \mathrm{~nm}$ ); insets are the photographs of the toluene gel under ambient light (left) and under illumination at $365 \mathrm{~nm}$ (right). (C) Emission and (D) UV-Vis absorption spectra of $\mathbf{1}$ in THF/water mixtures with different water volume fractions. The inset shows the changes in the emission intensity at $458 \mathrm{~nm}$ as a function of water fraction ([1] $\left.=1 \times 10^{-5} \mathrm{M}\right)$.

tested solvents except in bromobenzene, where though it behaves as the best gelator among the three species. The morphologies of these cyanostilbene-based organogels in toluene were studied by scanning electron microscopy (SEM) and transmission electron microscopy (TEM). Both microscopy techniques reveal gelator molecules aggregated into nano-fibrils of several microns in length, with a very high aspect ratio and with diameters ranging from 8 to 29 nm, respectively, as shown in Fig. 1B (and Fig. S7, ESI†).

Compounds $1-3^{9}$ are not soluble in several common polar and weakly polar solvents ( $\mathrm{MeOH}, \mathrm{MeCN}$, acetone, $\mathrm{CH}_{2} \mathrm{Cl}_{2}$ and $\mathrm{CHCl}_{3}$, EtOAc and DCE), while when dissolved in dioxane and THF, they are practically non-emissive. However, their fluorescence is distinctly enhanced upon addition of an increasing amount of water. For example in THF, as shown in Fig. 1C, 1 presents a weak band in emission at $429 \mathrm{~nm}$, whose intensity increases dramatically upon increasing the water molar fraction $\left(f_{\mathrm{w}}\right)$. In addition, a significant redshift of the emission occurs $(\Delta=29 \mathrm{~nm})$. A similar behaviour can be observed for compounds 2 and 3 (Fig. S8 and S9, ESI $†$ ).

As far as UV-Vis absorption is concerned, the UV-Vis absorption band of 1 is red-shifted from $c a .350 \mathrm{~nm}$, in pure THF solution, to ca. $400 \mathrm{~nm}$ upon increasing the water molar fraction $\left(f_{\mathrm{w}}\right)$ along with a gradual decrease in the intensity (Fig. 1D). This clearly indicates that the environment can affect the aggregation of 1-3, and these data are consistent with cyanostilbene moieties stacking together in J-type arrays. ${ }^{10}$ As compared to our previous report, ${ }^{7 b}$ where no obvious AIE was observed for a cyanostilbene derivative with a $\mathrm{NO}_{2}$ group in various solvents, it is apparent that the alkyl chains in 1-3 significantly promote the aggregation.

Notably, in line with the spectroscopic studies above, the gel of 1 in toluene exhibits a significantly enhanced emission (Fig. 2A), in contrast to the weak emission in solution. In addition, the gel of 1 in toluene emitted blue light with an emission maximum at

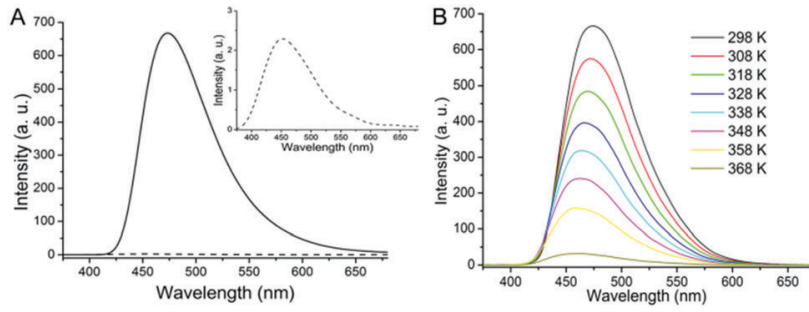

Fig. 2 (A) Fluorescence spectra of 1 in toluene solution (dashed line, $1 \times 10^{-5} \mathrm{M}$ ) and in the gel state (solid line, $5.7 \mathrm{mM}$ ), $\lambda_{\mathrm{ex}}=365 \mathrm{~nm}$. The inset shows the enlarged fluorescence spectrum of 1 in solution. (B) Temperaturedependent fluorescence spectra of gel 1 .

$475 \mathrm{~nm}$, redshifted by $c a .23 \mathrm{~nm}$ from the weak emission at $452 \mathrm{~nm}$ observed in dilute solution. Significant bathochromic shifts are commonly observed in aggregating systems, or in the solid state compared to solution, and point to strong interactions between gelator molecules in the gel state. Notably, when the toluene gel is heated until the solution state is reached, the emission peak intensity at $475 \mathrm{~nm}$ is greatly reduced (Fig. 2B), while emission is restored upon gel formation by cooling to r.t. As to the mechanism of AIE, it is quite established that the cyanostilbene moiety is practically non-fluorescent in the molecular solution state, where non-emissive relaxation can easily occur due to rotational freedom. Upon aggregation, such conformational motions are restricted, the molecule is planarized and, thus, relaxation goes through an emissive pathway.

Further studies were then aimed at a better elucidation of the driving forces responsible for gelation. Temperature-dependent ${ }^{1} \mathrm{H}$-NMR measurements were conducted on the gel of $\mathbf{1}$ in toluene (Fig. S10, ESI $\dagger$ ). Upon heating to $328 \mathrm{~K}$ no significant disaggregation of the networks into dissolved, NMR-observable aggregates occurred. Upon further heating, sharper signals appeared gradually; however, negligible shifts, also for the amidic NH group, were observed. Different is the case when a small aliquot of polar solvent is added to the gel sample. For example, in another experiment, $5 \mu \mathrm{L}$ DMSO- $d_{6}$ was added to the previous toluene gel 1 (initial gel volume $=1.0 \mathrm{~mL}$ ) and temperature-dependent ${ }^{1} \mathrm{H}$-NMR spectra were again recorded (Fig. S11, ESI $\dagger$ ). The addition of the small amount of DMSO causes notable changes. First of all, at $298 \mathrm{~K}$ the NH signal appears at $c a$. $10.44 \mathrm{ppm}$, a considerably downfield shifted value in comparison with the $7.90 \mathrm{ppm}$ signal seen in pure toluene. Also, under co-solvent conditions, gelator signals become more evident and less broadened, but most importantly, upon increasing $T$, a dramatic upfield shift in the amide protons (from 10.44 to $9.49 \mathrm{ppm})$ is observed. This large temperature dependence of amide protons $\left(\Delta \delta \mathrm{NH} / \Delta T=13.6 \times 10^{-3} \mathrm{ppm} \mathrm{K}^{-1}\right)$, not observed in the gel sample made in pure toluene, can be rationalized by a progressive disruption of the intermolecular hydrogen bonds (HBs) between DMSO and 1 molecules. If that is the case, this points to a minor contribution of HBs to the overall stabilization of the gel.

Finally, we analyzed toluene gel 1 by the FT-IR technique. Selected regions of the temperature-dependent FT-IR measurements for organogel 1 in toluene are shown in Fig. S12 (ESI $\dagger$ ). The band at $3325 \mathrm{~cm}^{-1}$, in the $\mathrm{N}-\mathrm{H}$ stretching region, can be 
assigned to the hydrogen-bonded $\mathrm{N}-\mathrm{H}$ stretching vibration, while the shoulder at $3440 \mathrm{~cm}^{-1}$ can be assigned to the free (not-bonded) $\mathrm{N}-\mathrm{H}$. Instead, the amide $\mathrm{I}$ band for $\mathrm{C}=\mathrm{O}$ appears at $1678 \mathrm{~cm}^{-1}$. Upon increasing the temperature, the hydrogenbonded $\mathrm{N}-\mathrm{H}$ band gradually decreases while the amide I band is blue-shifted to $1691 \mathrm{~cm}^{-1}$ ( ca. $13 \mathrm{~cm}^{-1}$ ), confirming the participation of the amide groups in the formation of hydrogen bonds in the organogel. Overall, aggregation of 1 can be viewed, no surprise about that, as the resultant of several different intermolecular interactions, with a major contribution of aromatic stacking, while HB interactions surely have a lower impact.

Cyanostilbene could be switched from the initial trans (Z-) form to the cis (E-) form upon UV light irradiation. However, compared to the more common stilbene and azobenzene molecules, the $Z-E$ isomerization process of cyanostilbene derivatives is little known and literature data are especially scarce regarding the corresponding fluorescence modulation in supramolecular gels. ${ }^{5 c}$ As compound $\mathbf{1}$ is a very good gelator, we were interested in verifying whether the gelator configuration would have an effect on its gelation properties or not. Thus, we first studied in more detail the light induced $Z$-to- $E$ isomerization process of 1 in solution by ${ }^{1} \mathrm{H}-\mathrm{NMR}$. In the initial state, the cyanostilbene unit in 1 adopts a $Z$ configuration, as evidenced by well-assigned ${ }^{1} \mathrm{H}$-NMR signals (Fig. S13, ESI $\dagger$ ). The $Z$-to- $E$ photoisomerization occurs when the compound is irradiated using UV light at $365 \mathrm{~nm}$. The appearance of new $\mathrm{NH}$ peaks corresponding to the formation of new isomers can be observed. As 1 possesses two photoisomerizable double bonds, the starting material in configuration $Z, Z$ can give rise to two different photoproducts, $E, Z$ - and $E, E$-isomers, all easily identifiable in the $\mathrm{NH}$ region (10.5-10.8 ppm). As seen in Fig. 3A, over a prolonged irradiation time, the amount of $Z, E$ - and $E, E$-isomer products increased gradually with a concomitant decrease of the $Z, Z$-isomer until a photostationary state is reached. By the integral analysis of the ${ }^{1} \mathrm{H}$-NMR spectra of $\mathbf{1}$ in DMSO- $d_{6}$ at different irradiation times kinetic profiles could be plotted (Fig. 3B). After $c a$. $1 \mathrm{~h}$ irradiation time, the photostationary state is composed of the following isomeric mixture: $Z, Z: Z, E: E, E$ ratio of $c a$. $7: 38: 55$. We then tested the response of the gel state to irradiation. Toluene gel 1 $\left(c_{\mathrm{tol}}=4.1 \mathrm{mM}\right)$ was irradiated with $365 \mathrm{~nm}$ light (Fig. 3C and D). After 40 minutes, as documented by photographs in Fig. 3D, the gel was transformed into a viscous solution. The obtained solution state was also analyzed by ${ }^{1} \mathrm{H}-\mathrm{NMR}$, confirming the presence of significant amounts of $Z, E$ and $E, E$ isomers (Fig. S14, $\mathrm{ESI} \dagger)$. As the temperature was maintained constant during irradiation, the gel-to-sol transition can be unambiguously considered to be photoinduced. Hence, it can be concluded that the $Z, Z$ isomer has a considerably stronger gelating ability than the other two isomers which are gradually formed by irradiation. Interestingly, the isomerization reaction can be reversed by heating (Fig. S14, ESI $\dagger$ ). Thus, if the irradiated solution is heated for a short time, it can be transformed back into a stable gel.

Toluene gel 1 was also found to be selectively responding to TFA exposure in a way which could be interesting for TFA sensing purposes (Fig. 4). Fluorescent gelators capable of acid sensing are rare. Moreover, in all the systems reported in the
A
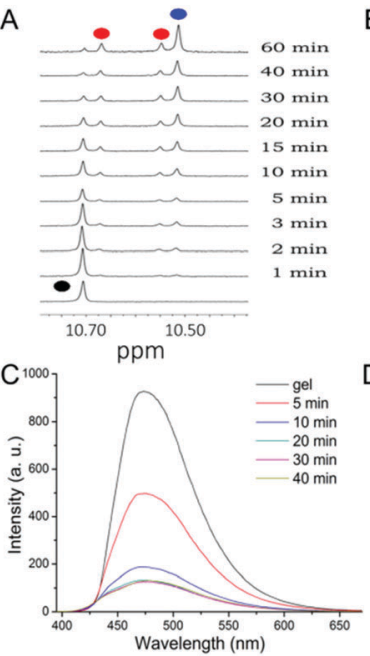

Fig. 3 (A) ${ }^{1} \mathrm{H}-\mathrm{NMR}$ spectral changes of 1 upon photoirradiation $(7 \mathrm{mM}$ in $\mathrm{DMSO}-d_{6}$ at $298 \mathrm{~K}$ ) starting with the $Z, Z$ isomer followed by irradiation with UV light (365 nm) at $298 \mathrm{~K}$. (B) Time evolution of the isomer ratio of 1 as estimated by integral analysis. (C) Time-dependent fluorescence spectra of toluene gel 1 upon irradiation with $365 \mathrm{~nm}$ light. (D) Photographs of toluene gel 1 after photoirradiation for (from left to right) 0, 5, 10, 20, 30 and 40 minutes under UV light.

literature so far, the gelator molecules contain clearly defined basic sites that can specifically interact with acidic species, resulting in shifts in emission wavelength or fluorescence quenching. ${ }^{8}$ The gel of $\mathbf{1}$ in toluene could be converted into fluid solution upon exposing to TFA vapors (Fig. 4C-b). Consequently, the emission at $475 \mathrm{~nm}$, as said to have been induced by aggregation, was also quenched immediately. Under the same conditions, other examined volatile acid vapors, such as $\mathrm{HCl}, \mathrm{HBr}$, formic acid and acetic acid, could not lead to gel decomposition and had no effects on the emission spectra of toluene gel 1 (Fig. 4A). Therefore, the gel possesses excellent selectivity for TFA. The same behaviour was also observed upon addition of a few drops of TFA on the top of the preformed gel

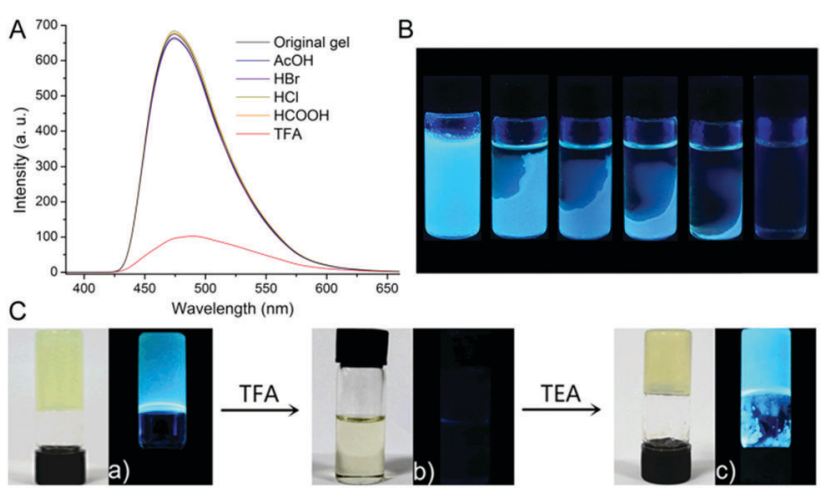

Fig. 4 (A) Fluorescence spectra of toluene gel $1(5.7 \mathrm{mM})$ upon its exposure to different acid vapors at $298 \mathrm{~K}\left(\lambda_{\mathrm{ex}}=365 \mathrm{~nm}\right)$; (B) response of the gel system to the addition of TFA drops onto its top layer over a period of a few minutes; (C) photographs under natural light (left) and under UV light (right) of (a) toluene gel $\mathbf{1}$, (b) the system after exposure to TFA vapors and (c) restoration of the gel state upon addition of TEA followed by sonication. 
(Fig. 4B), while, again, other acids did not perturb the gel phase. This clearly highlights the selective response of the gel towards TFA over other common acidic species. The gel sample transformed to solution upon exposure to TFA vapors was analyzed by ${ }^{19} \mathrm{~F}$-NMR in order to determine the actual amount of acid needed to produce such a conspicuous transition (Fig. S15, ESI $\dagger$ ). Interestingly, the amount of TFA calculated by integral analysis in the presence of an internal standard of known concentration was found to be quite high, $c a$. 75 times the gelator molecule in terms of molar ratio. On the other hand, the ${ }^{1} \mathrm{H}-\mathrm{NMR}$ spectrum recorded on the same sample reveals small downfield shifts of the aromatic proton signal (Fig. S16, ESI $\dagger$ ). A more accurate titration experiment of compound 1 with TFA in DMSO solvent confirms that no conspicuous changes in the NMR spectrum of $\mathbf{1}$ are present, indicating that no evident protonation occurs (Fig. S17, ESI $\dagger$ ). This is an important feature which distinguishes this system with respect to others, ${ }^{8}$ as the gel to solution transition cannot be attributed to an acid-base reaction, despite the strong acidic nature of TFA. In contrast, we believe that the effect of TFA, which cannot be observed with other acids, is due to its more liphophilic character. In other words, TFA is capable of being adsorbed on the toluene gel made of $\mathbf{1}$ disrupting gelator-gelator interactions, especially the stacking which is a key element to gel formation.

Finally, the reversibility of the gel was also examined since this feature can be a determinant for potential applications of this system as a selective chemosensor. Thus, the solution state sample was exposed to triethylamine (TEA) vapors for a short time, and indeed, the original gel could be restored, as also proven by the reappearance of the blue fluorescence (Fig. 4C-c). No signs of degradation of 1 were observed. Notably, the TFA-TEA cycle can be repeated more than three times, thus showing the potential of this system for fluorimetric sensing of acid vapors.

In summary, we have prepared a novel stimuli-responsive $\pi$-conjugated organogelator 1 which is capable of forming AIE fluorescent gels in aromatic solvents at low concentration. The system shows interesting bi-responsive properties. Indeed, the organogel exhibits photoresponsive behavior as the gel breaks upon exposure to UV irradiation at $365 \mathrm{~nm}$. The mechanism responsible for such a gel-breaking phenomenon is clearly the cis-trans isomerization of the cyanostilbene moiety, as confirmed by ${ }^{1} \mathrm{H}$-NMR spectroscopy. Furthermore, fumigation of toluene gel 1 with TFA-TEA vapors exhibits an off/on switching between the gel and the solution state, with the consequent fluorescence quenching/restoration, a cycle that can be repeated several times. The response to acidic TFA is found to be selective, as the exposure of the gel sample to other acid vapors (such as $\mathrm{HCl}, \mathrm{HBr}$, formic acid and acetic acid) does not induce any significant effect. Interestingly, the TFA-gelator interaction cannot be attributed to a specific acid-base reaction, in contrast to other examples of acid responsive gels. Rather, we speculate that TFA is the only acid, among those tested, whose vapors can be easily dissolved in the gel, thus leading to disaggregation and the solution state. We believe that the design strategy toward multistimuli-responsive fluorescence switching materials applied here may be widely applicable to the development of other functional materials, and further studies along this research line are currently in progress in our laboratories.

This work was supported by the National Natural Science Foundation of China (21374036), the National Basic Research Program of China (2012CB933800) and by a Croatian-Chinese bilateral project.

\section{Notes and references}

1 (a) B. O. Okesola and D. K. Smith, Chem. Soc. Rev., 2016, 45, 4226; (b) M. Cametti and Z. Džolić, Chem. Commun., 2014, 50, 8273; (c) R. G. Weiss, J. Am. Chem. Soc., 2014, 136, 7519; (d) J. W. Steed, Chem. Commun., 2011, 47, 1379; (e) Y. Zhang, Y. Ma, M. Deng, H. Shang, C. Liang and S. Jiang, Soft Matter, 2015, 11, 6162; $(f)$ S. Dong, Y. Luo, X. Yan, B. Zheng, X. Ding, Y. Yu, Z. Ma, Q. Zhao and F. Huang, Angew. Chem., Int. Ed., 2011, 50, 1905; (g) X. Yan, D. Xu, X. Chi, J. Chen, S. Dong, X. Ding, Y. Yu and F. Huang, Adv. Mater., 2012, 24, 362; (h) M. Zhang, D. Xu, X. Yan, J. Chen, S. Dong, B. Zheng and F. Huang, Angew. Chem., Int. Ed., 2012, 51, 7011.

2 (a) Molecular Gels Materials with Self-Assembled Fibrillar Networks, ed. R. G. Weiss and P. Terech, Springer, Dordrecht, Netherlands, 2006; (b) Functional Molecular Gels, ed. B. Escuder and J. F. Miravet, RSC, Cambridge, 2014.

3 (a) X. Yu, L. Chen, M. Zhang and T. Yi, Chem. Soc. Rev., 2014, 43, 5346; (b) C. Ren, J. Zhang, M. Chen and Z. Yang, Chem. Soc. Rev., 2014, 43, 7257; (c) C. D. Jones and J. W. Steed, Chem. Soc. Rev., 2016, 45, DOI: 10.1039/C6CS00435K.

4 (a) J. Majumder and P. Dastidar, Chem. - Eur. J., 2016, 22, 9267; (b) M. Yamanaka, K. Yanai, Y. Zama, J. Tsuchiyagaito, M. Yoshida, A. Ishii and M. Hasegawa, Chem. - Asian J., 2015, 10, 1299; (c) X. Yu, L. Chen, M. Zhanga and T. Yi, Chem. Soc. Rev., 2013, 42, 7086; (d) Z. Džolić, M. Cametti, D. Milić and M. Žinic, Chem. - Eur. J., 2013, 19, 5411; (e) Z. Džolić, M. Cametti, A. Dalla Cort, L. Mandolini and M. Žinić, Chem. Commun., 2007, 3535; $(f)$ L. Zang, H. Shang, D. Wei and S. Jiang, Sens. Actuators, B, 2013, 185, 389.

5 (a) E. R. Draper and D. J. Adams, Chem. Commun., 2016, 52, 8196; (b) S. J. Wezenberg, C. M. Croisetu, M. C. A. Stuarta and B. L. Feringa, Chem. Sci., 2016, 7, 4341; (c) J. Seo, J. W. Chung, J. E. Kwon and S. Y. Park, Chem. Sci., 2014, 5, 4845.

6 (a) S. S. Babu, V. K. Praveen and A. Ajayaghosh, Chem. Rev., 2014, 114, 1973; (b) R. Hu, N. L. C. Leung and B. Z. Tang, Chem. Soc. Rev., 2014, 43, 4494; (c) Z. Zhao, J. W. Y. Lamb and B. Z. Tang, Soft Matter, 2013, 9, 4564; (d) J. Mei, N. L. C. Leung, R. T. K. Kwok, J. W. Y. Lam and B. Z. Tang, Chem. Rev., 2015, 115, 11718.

7 (a) Y. Zhang, C. Liang, H. Shang, Y. Ma and S. Jiang, J. Mater. Chem. C, 2013, 1, 4472; (b) Y. Zhang and S. Jiang, Org. Biomol. Chem., 2012, 10, 6973.

8 (a) J. Peng, J. Sun, P. Gong, P. Xue, Z. Zhang, G. Zhang and R. Lu, Chem. - Asian J., 2015, 10, 1717; (b) P. Xue, B. Yao, P. Wang, P. Gong, Z. Zhang and R. Lu, Chem. - Eur. J., 2015, 21, 17508; (c) J. Sun, P. Xue, J. Sun, P. Gong, P. Wang and 
R. Lu, J. Mater. Chem. C, 2015, 3, 8888; (d) X. Qian, W. Gong, M. K. Dhinakaran, P. Gao, D. Na and G. Ning, Soft Matter, 2015, 11, 9179; (e) P. Xue, R. Lu, P. Zhang, J. Jia, Q. Xu, T. Zhang, M. Takafuji and H. Ihara, Langmuir, 2013, 29, 417. 9 Compounds 1-3 were synthesized in good yield by condensation of 5-alkoxy-isophthaloyl dichlorides with 2-phenyl-3-( $p$-aminophenyl)acrylonitrile in the presence of triethylamine. In DMSO- $d_{6}$, their NMR spectra are in agreement with their structures with the $C 2$ axis.

10 (a) B.-K. An, S.-K. Kwon, S.-D. Jung and S. Y. Park, J. Am. Chem. Soc., 2002, 124, 14410; (b) P. Xing, H. Chen, L. Bai and Y. Zhao, Chem. Commun., 2015, 51, 9309. 